\title{
Broadband VSF-OFCDM Systems with Hybrid MCI Cancellation and MMSE Detection
}

\author{
Y.Q. Zhou and J. Wang \\ Dept. of Electrical and Electronic Engineering \\ The University of Hong Kong, HONG KONG \\ yqzhou@eee.hku.hk
}

\begin{abstract}
In this paper, an analytical study is presented on the performance of the variable spreading factor orthogonal frequency and code division multiplexing (VSF-OFCDM) system with hybrid multi-code interference (MCI) cancellation and minimum mean square error (MMSE) detection. The weights of MMSE are derived, and should be updated stage by stage of MCI cancellation. Various values of time and frequency domain spreading factors are dealt with in a QPSK-modulated VSFOFCDM system. It is shown that the hybrid detection scheme performs much better than the conventional MMSE. For the hybrid detection, a larger value of the frequency domain spreading factor is expected.
\end{abstract}

\section{INTRODUCTION}

A huge bandwidth is needed for future wireless systems to transmit high-speed data. It has been shown in [1] that in a broadband channel, orthogonal frequency and code division multiplexing (OFCDM) exhibits better performance than direct sequence CDMA (DS-CDMA) scheme. This is because OFCDM is based on orthogonal frequency division multiplexing (OFDM), and can combat the multipath interference. Moreover, to work in various cell environments, two-dimensional variable spreading factor (VSF) is proposed for the OFCDM system [2]. The total spreading factor $(N)$ is the product of time domain spreading factor $\left(N_{T}\right)$ and frequency domain spreading factor $\left(N_{F}\right)$, i.e., $N=N_{T} \times N_{F}$, where $N, N_{T}$ and $N_{F}$ can be changed flexibly to provide desired quality of service (QoS) according to different conditions.

Multi-code transmission is one of efficient ways to achieve high-speed transmission [3]. However, in a realistic mobile channel, the orthogonality between multi-code channels no longer maintains in time domain because of possible fast fading or in frequency domain because of independent fading among sub-carriers. Since a short packet length is preferred in high-speed packet transmission, the channel variation in one packet duration is negligible in most cases. Hence, time domain spreading can preserve the orthogonality between code channels. On the other hand, the orthogonality between code channels in frequency domain will be distorted by the frequency selectivity of broadband channels, so that multi-code interference $(\mathrm{MCl})$ may occur. In order to improve performance, $\mathrm{MCI}$ must be cancelled out as much as possible. Thus, it is desirable to evaluate the performance of the VSFOFCDM system when MCl cancellation technique is adopted.

The OFCDM system with only frequency domain spreading has been well studied in [4-5]. Various detection (or combining) techniques have been proposed, such as maximum ratio combining (MRC), minimum mean square error (MMSE) and maximum likelihood detection (MLD). MLD is optimum but its complexity increases exponentially with the number of code channels. MMSE has been widely studied [6]. However, interference cancellation technique has not been investigated yet for the VSF-OFCDM system. The objective of this paper is to analytically investigate the performance of VSF-OFCDM with hybrid MCI cancellation and MMSE detection.

The rest of the paper is organized as follows. Section 2 introduces the system model. Section 3 presents pure MMSE detection and hybrid $\mathrm{MCI}$ cancellation and MMSE detection. The error probability is discussed in Section 4. Section 5 presents some representative numerical results. Finally, Section 6 draws conclusions.

\section{SYSTEM MODEL}

\section{A. System Description}

The block diagram of the VSF-OFCDM system is shown in Fig. 1. At the transmitter (Fig. I(a)), information bits on each data channel are firstly processed by a QPSK mapper with Gray coding. QPSK symbols are serial-to-parallel (S/P) converted into $M / N_{F}$ streams, where $M$ is the total number of sub-carriers. Every symbol of the streams will be twodimensionally spread. Scrambling will be carried out on the spread chips. After spreading and scrambling, all code channels are combined at code multiplexer. A frequency interleaver is then employed to provide frequency diversity. After interleaving, totally $M$ chips should be up-converted to $M$ subcarriers and transmitted in parallel. An $M$-point IFFT realizes this operation. At the output of IFFT, an effective OFCDM symbol with duration $T_{s}$ is obtained. Then a guard interval is inserted between OFCDM symbols to prevent intersymbol interference (ISI). Finally, the complete OFCDM symbol with duration $T$ passes through pulse shaping filter, are up-converted to carrier frequency and transmitted.

At receiver, signals are firstly down-converted to baseband signals. With recovered OFCDM symbol timing information, signals are processed by a matched filter. The resultant signals are further input to FFT block, which realizes the $M$-sub-carrier down conversion. After FFT, the $M$ chips are obtained and deinterleavered. Then time domain despreading is carried out. When the MCI cancellor is employed, the output from time domain despreader will be subtracted by regenerated $\mathrm{MCI}$. The remainders are then multiplied by weights obtained from MMSE algorithms and combined at. the frequency domain 
despreader. After despreading, a hard decision is used to recover QPSK symbols. Then signals are parallel-to-serial (P/S) converted and demapped. Finally, information bits are obtained, which will be used to regenerate the $\mathrm{MCI}$ for other code channels. Similarly, the recovered information bits from other data channels are also used to regenerate the MCI for current data channel. Basically, interference regenerator performs the operation as the transmitter except channel information. The cancellation process will continue in an iterative way until the specified number of stages is reached. The MMSE weights must be updated stage by stage due to the reduction of the $\mathrm{MCI}$ in each stage. Generally speaking, as the recovered information bits become more reliable stage by stage, $\mathrm{MCI}$ can be regenerated with higher accuracy, After subtraction, $\mathrm{MCI}$ can be cancelled out much from the received signal. Thus the bit error rate (BER) performance can be improved stage by stage.

\section{B. Two-dimensional Spreading Sequences}

As defined before, the total spreading factor of twodimensional spreading is expressed as $N=N_{T} \times N_{F}$. The time domain OVSF spreading code of length $N_{T}$ is denoted as $C_{N_{T}}^{\left(k_{T}\right)}=\left\{c_{N_{T}, 0}^{\left(k_{T}\right)}, \cdots, c_{N_{T}, N_{T}-1}^{\left(k_{T}\right)}\right\}$, and the frequency domain OVSF spreading code of length $N_{F}$ is denoted as $C_{N_{F}}^{\left(k_{F}\right)}=\left\{c_{N_{F}, 0}^{\left(k_{F}\right)}, \cdots, c_{N_{F}, N_{F}-1}^{\left(k_{F}\right)}\right\}$. Suppose that the $k^{\text {th }}$ code channel employs the two-dimensional spreading code $\left\{C_{N_{T}}^{\left(k_{T}\right)}, C_{N_{F}}^{\left(k_{F}\right)}\right\}$. If $K$ is the total number of code channels, for the $k^{\text {th }}$ code channel, the code set of other $K-1$ code channels can be divided into two sub-sets:

$$
\begin{gathered}
\Omega_{d}=\left\{C_{N_{T}}^{\left(k_{T}\right)}, C_{N_{F}}^{\left(\hat{k}_{r}\right)}\right\}, \hat{k}_{F}=0, \cdots, N_{F}-1, b u t \quad \hat{k}_{F} \neq k_{F} \\
\Omega_{B}=\left\{C_{N_{F}}^{\left(\hat{k}_{T}\right)}, C_{N_{F}}^{\left(\hat{k}_{F}\right)}\right\}, \hat{k}_{F}=0, \cdots, N_{F}-1, \hat{k}_{T}=0, \cdots, N_{T}-1, \text { but } \hat{k}_{T} \neq k_{T}
\end{gathered}
$$

In highly frequency selective channels, code channels from $\Omega_{A}$ cause severe MCI to the $k^{\text {th }}$ code channel because their othogonality in frequency domain is distorted by independent fading on sub-carriers. On the other hand, in a slow fading channel or in a short packet, the orthogonality in time domain between any code channel from $\Omega_{B}$ and the $k^{\text {th }}$ code channel is maintained. Thus, there is no MCI from $\Omega_{B}$ and the number of effective interference code channels is equal to the number of code channels in $\Omega_{A}$, which is defined as $K_{C}$. To keep $\mathrm{MCI}$ small for each code channel, time domain spreading codes should be assigned first. Therefore, for $K$ code channels, the number of interfering code channels in for each code channel will be at most: $K_{C}=\left\lfloor(K-1) / N_{\tau}\right\rfloor$, where $\lfloor x\rfloor$ is the integer portion of $x$.

\section{DECISION VARIABLES}

On downlink, the transmitted signal of the $m^{\text {th }}$ sub-carrier on the $i^{\text {th }}$ chip can be expressed as:

$$
S_{m, i}(t)=\sqrt{P} \sum_{k=0}^{K-1} a_{k, m, i} e^{j 2 \pi f_{m}(t-i T)} p(t-i T)
$$

where $P$ is the signal power of one code on one sub-carrier, and $a_{k, m, i}=d_{k} \cdot c_{N_{T}, i}^{\left(k_{T}\right)} \cdot c_{N_{F}, m}^{\left(k_{F}\right)} \cdot s_{m, i}$. Here $d_{k}$ is the data symbol of $k^{\text {th }}$ code channel, $s_{m, i}$ is the $i^{\text {th }}$ chip on $m^{\text {th }}$ sub-carrier of the random binary scrambling code, and $f_{m}=m / T_{s}$ is the baseband equivalent frequency of $m^{\text {th }}$ sub-carrier. $p(t)$ is assumed to be a rectangular pulse with unity amplitude and duration of $T$.

As described before, each code channel is involved with $N_{F}$ separate (or interleaved) sub-carriers in $N_{T}$ chips' duration. However, for simplicity of notation, frequency interleaving is omitted and thus only the first $N_{F}$ sub-carriers are dealt with.

\section{A. Received Signal}

It is assumed that the signal of the OFCDM system transmitted on each sub-carrier experiences a flat fading channel with fixed fading over $N_{T}$ OFCDM symbols. Furthermore, with frequency interleaving, it is reasonable to assume that all the $N_{F}$ sub-carriers experience independent fading. Thus, the received signal at the $\hat{m}^{\text {th }}$ sub-carrier on the $\hat{i}^{\text {th }}$ chip can be expressed as:

$$
r_{\hat{m}, i}(t)=h_{\hat{m}} \cdot S_{\hat{m}, \hat{i}}(t)+\eta_{\hat{m}, \hat{i}}(t)
$$

where $h_{m}$ is the complex channel fading for the $\hat{m}^{\text {th }}$ subcarrier with $E\left\{\left|h_{\hat{m}}\right|^{2}\right\}=1$ and $\eta_{\bar{m}, \hat{i}}(t)$ is a complex Gaussian noise on the $\hat{m}^{\text {th }}$ sub-carrier with double-sided power spectrum density of $N_{0}$. Both $h_{\hat{m}}$ and $\eta_{\hat{m}, \hat{i}}(t)$ are assumed to be independent. With ideal symbol timing, this baseband signal inputs to the matched filter. By setting guard interval larger than the maximum channel delay, there is no interference from adjacent OFCDM symbols. If the $0^{\text {th }}$ code with twodimensional spreading code $\left\{C_{N_{T}}^{(0)}, C_{N_{F}}^{(0)}\right\}$ is the desired code, then time domain despreading is carried out, with output:

$$
\begin{aligned}
r_{0}(\hat{m}) & =\frac{1}{N_{T} \cdot T_{s}} \sum_{i=0}^{N_{T}-1} \int_{T}^{T+T_{s}} r_{\tilde{m}_{i}, i}(t) e^{-j 2 \pi f_{\hat{m}}(t-i \cdot T)} p\left(T_{s}-t+\hat{i} \cdot T\right) d t \cdot c_{N_{t}, \hat{i}}^{(0)} \cdot s_{\hat{m}, \hat{i}} \\
& =S_{T, 0}(\hat{m})+I_{T, 0}(\hat{m})+N_{T, 0}(\hat{m})
\end{aligned}
$$

where $S_{T, 0}(\hat{m})$ is the useful signal component and $I_{T, 0}(\hat{m})$ is the interference from other $K_{C}$ interference codes, and $N_{T, 0}(\hat{m})$ is the background noise with the variance of $\sigma_{T, n}^{2}=\sigma_{n}^{2} / N_{T}=N_{0} /\left(N_{T} \cdot T_{s}\right)$.

\section{B. Pure MMSE Detection}

At the $0^{\text {th }}$ stage of $\mathrm{MCI}$ cancellation, the regenerated $\mathrm{MCl}$ is unavailable. Thus pure MMSE detection is used to combine the useful signals from different sub-carriers. In this case, the total power of data signals input to MMSE detector is $P_{\text {total }}=\left(K_{c}+1\right) P$. As well-known, the weights of MMSE are given by [6]: 


$$
\omega(\hat{m})=\frac{\dot{h_{m}}}{\left|h_{\dot{m}}\right|^{2}+\left(N_{T}\left(1+K_{c}\right) \frac{E_{s}}{N_{0}}\right)^{-1}}
$$

where $E_{s} / N_{0}=P T_{s} / N_{0}$. Thus, the output of the frequency domain despreader is given by:

$$
y_{0}=\frac{1}{N_{F}} \sum_{m=0}^{N_{F}-1} r_{0}(\hat{m}) \cdot\left[c_{N_{F}, \hat{m}}^{(0)} \cdot \omega(\hat{m})\right]
$$

With ideal estimation of channel fading, signal power and noise variance, a hard decision is used on $y_{0}$ to recover the data on the $0^{\text {th }}$ code channel.

\section{Hybrid MCI cancellation and MMSE Detection}

With tentative data decisions $\hat{d}_{k_{F}}^{(s-1)}$ of the $(s-1)^{\text {th }}$ stage and ideal channel estimation $h_{\hat{m}}, \mathrm{MCl}$ in the $s^{\text {th }}$ stage can be regenerated for the $\widehat{m}^{\text {th }}$ sub-carrier of the $0^{\text {th }}$ code channel:

$$
\hat{I}(s),(\hat{m})=\sqrt{P} \cdot h_{\hat{m}} \cdot \sum_{k_{F}=1}^{K_{c}} \hat{d}_{k_{F} \cdot N_{T}}^{(s-1)} \cdot c_{N_{F}, \hat{m}}^{\left(k_{F}\right)}
$$

The signal after MCI cancellation is given by:

$$
r_{0}^{(s)}(\hat{m})=S_{T, 0}(\hat{m})+I_{T, 0}^{(s)}(\hat{m})+N_{T, 0}(\hat{m})
$$

where $I_{t .0}^{(s)}(\hat{m})$ is the residual MCI after $s^{\text {th }}$ stage of cancellation, given by:

$$
\begin{aligned}
I_{T, 0}^{(s)}(\hat{m}) & =I_{T, 0}(\hat{m})-\hat{I}_{T, 0}^{(s)}(\hat{m}) \\
& =\sqrt{P} \cdot h_{\hat{m}} \cdot \sum_{k_{F}=1}^{K_{c}}\left(d_{k_{F}: *_{T}}-\hat{d}_{k_{F}, N_{F}}^{(s-1)}\right) \cdot c_{N_{F}, \hat{m}}^{\left(k_{F}\right)}
\end{aligned}
$$

When QPSK with Gray mapping is employed, the variance of the residual $\mathrm{MCl}$ on $s^{\text {th }}$ stage is given by:

$$
\sigma_{I}^{2}(s)=P \cdot K_{C} \cdot E\left\{\left|d_{k_{F} \cdot N_{T}}-\hat{d}_{k_{F} \cdot N_{F}}^{(s-1)}\right|^{2}\right\}=4 P \cdot K_{C} \cdot P_{b}^{(s-1)}
$$

Therefore, the new weights of MMSE with MCl cancellation can be expressed as:

$$
\omega^{(s)}(\hat{m})=\frac{h_{\hat{m}}^{*}}{\left|h_{\hat{m}}\right|^{2}+\left(N_{T}\left(1+4 K_{C} P_{b}^{(s-1)}\right) \frac{E_{s}}{N_{0}}\right)^{-1}}
$$

Finally, the decision variable for the data signal on $0^{\text {th }}$ code channel after hybrid MCI cancellation and MMSE detection is expressed as:

$$
\begin{aligned}
y_{0}^{(s)} & =\frac{1}{N_{F}} \sum_{m=0}^{N_{F}-1} r_{0}^{(s)}(\hat{m}) \cdot\left[c_{N_{F}, \hat{m}}^{(0)} \cdot \omega^{(s)}(\hat{m})\right] \\
& =S_{0}^{(s)}+M C I_{0}^{(s)}+\eta_{0}^{(s)}
\end{aligned}
$$

where $S_{0}^{(s)}$ is the desired signal component, $M C I_{0}^{(s)}$ is the residual $\mathrm{MCI}$, and $\eta_{0}^{\langle s\rangle}$ is the background noise component. Note that with hybrid detection, not only $\mathrm{MCl}$ but also useful signal and background noise vary with stages because of MMSE weight updating.

\section{PERformance EVAluation}

Given $\left\{h_{\dot{m}}\right\},\left\{c_{N_{T}, i}^{(0)}\right\}$ and $\left\{c_{N_{F}, m}^{(0)}\right\}$, the error probability of the real and imaginary parts of decision variable $y_{0}^{(s)}$ should be equal. The real part, $y_{l, 0}^{(s)}=S_{l, 0}^{(s)}+M C I_{l, 0}^{(s)}+\eta_{l, 0}^{(s)}$, is considered, where $S_{I, 0}^{(s)}=A^{(s)} \cdot d_{I, 0}$ is the real part of the desired component, $d_{i, 0}$ is the real part of desired data, and $A^{(s)}=\sqrt{P /\left(2 N_{F}^{2}\right)} \cdot \sum_{m=0}^{N_{F}-1} h_{\hat{m}} \cdot \omega^{(s)}(\hat{m})$ is the desired amplitude. Furthermore, $M C I_{I, 0}^{(s)}$ is the real part of the residual $\mathrm{MCI}$, given by:

$$
M C I_{l, 0}^{(s)}=\sqrt{\frac{P}{2}} \cdot \frac{1}{N_{F}} \cdot \sum_{k_{F}=1}^{K_{C}}\left(\begin{array}{l}
\left(2 d_{1, k_{f} \cdot N_{I}} \cdot e_{k_{F}}^{(s-1)}\right) \cdot \\
\sum_{\hat{m}=0}^{N_{F}-1} h_{\hat{m}} \cdot\left[c_{N_{F}, \hat{m}}^{(0)} \cdot c_{N_{F}, \hat{n} t}^{\left(k_{F}\right)} \cdot \omega^{(s)}(\hat{m})\right]
\end{array}\right)
$$

where $d_{t, k_{F} \cdot N_{T}}$ is the real part of the data and $e_{k_{F}}^{(s-1)}$ stands for the decision error. When $e_{k_{F}}^{(s-1)}=0$, there is no data error. However, when $e_{k_{F}}^{(s-1)}=1$, there is a bit error on the $\left(k_{F} \cdot N_{T}\right)^{\text {th }}$ code. The probability of $e_{k_{f}}^{(s-1)}=0$ and 1 are given by $P\left(e_{k_{f^{\prime}}}^{(s-1)}=0\right)=1-P_{b}^{(s-1)}$ and $P\left(e_{k_{f}}^{(s-1)}=1\right)=P_{b}^{(s-1)}$, respectively. Finally, $\eta_{i, 0}^{(s)}$ is the real part of the channel noise component with variance $\sigma_{n_{t, 0}^{(s)}}^{2}=\frac{\sigma_{n}^{2}}{2 N \cdot N_{F}} \sum_{m=0}^{N_{F}-1}\left|\omega^{(s)}(\hat{m})\right|^{2}$.

Given $\left\{h_{\hat{m}}\right\},\left\{d_{I, k_{F} \cdot N_{T}}\right\}$ and $\left\{e_{k_{F}}^{(s-1)}\right\}$, the conditional error probability is given by:

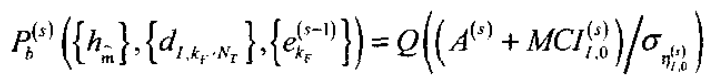

where $Q(z)=(1 / \sqrt{2 \pi}) \int_{z}^{+\infty} e^{\frac{t^{2}}{2}} d t$ for $z \geq 0$.

In order to obtain the average bit error rate, (15) should be averaged over all conditions. Define $\left\{e_{k_{F}}^{(s-1)}\right\}_{n, i}, i=1, \cdots,\left(\begin{array}{l}K_{C} \\ n\end{array}\right)$, as the error vector with $n$ bit errors and $i^{\text {th }}$ order, where $\left(\begin{array}{l}K_{C} \\ n\end{array}\right)=\frac{K_{C} !}{n !\left(K_{C}-n\right) !}$. Given $\left\{e_{k_{F}}^{(s-1)}\right\}_{n, i}$, only $n$ out of $K_{C}$ data can contribute to $M C I_{l, 0}^{(s)}$, because remaining $K_{C}-n$ data are cancelled out by correct tentative decisions. Therefore, only $2^{n}$ 
data combinations $\left(\left\{d_{l \cdot k_{F} \cdot N_{T}}\right\}_{n, i, j}, j=1,2, \cdots, 2^{n}\right)$ should be accounted for in the evaluation of $M C I_{i .0}^{(s)}$. Since each data combination is taken with equal probability $2^{-n}$, conditioned on $\left\{e_{k_{r}}^{(s-1)}\right\}_{n, i}$, the average of (15) over $\left\{d_{I, k_{F} \cdot N_{I}}\right\}$ is given by:

$$
\begin{aligned}
& P_{b}^{(s)}\left(\left\{h_{m}\right\},\left\{e_{k_{F}}^{(s-1)}\right\}_{n, i}\right) \\
& =\sum_{j=1}^{2^{n}} 2^{-n} \cdot P_{b}^{(s)}\left(\left\{h_{\hat{m}}\right\},\left\{d_{l, k_{F} \cdot N_{T}}\right\}_{n, i, j},\left\{e_{k_{F}}^{(s-1)}\right\}_{n, i}\right)
\end{aligned}
$$

For a specific error vector $\left\{e_{k_{F}}^{(s-1)}\right\}_{n, i}$, the probability of $n$ bit errors out of $K_{C}$ codes is given by

$$
P(n)=\left(P_{b}^{(s-1)}\right)^{n} \cdot\left(1-\dot{P}_{b}^{(s-1)}\right)^{K_{c}-n}
$$

When $n$ is large, (17) is very small. Thus, in numerical calculation, only a few small values of $n$ need to be considered. Therefore, the average of (16) over $\left\{e_{k_{F}}^{(s-1)}\right\}_{n, i}$ is given by:

$$
P_{b}^{(s)}\left(\left\{h_{m}\right\}\right)=\sum_{n=0}^{K_{c}} P(n) \sum_{i=1}^{\left(\begin{array}{l}
K_{c} \\
n
\end{array}\right)} P_{b}^{(s)}\left(\left\{h_{m}\right\},\left\{e_{k_{F}}^{(s-1)}\right\}_{n, i}\right)
$$

In the special case (i.e. $0^{\text {th }}$ stage or pure MMSE detection), regenerated $\mathrm{MCI}$ does not exist or $\left\{e_{k_{F}}^{(s-t)}\right\}$ is equal to a vector with all elements valued "l" in (14). Both (17) and (18) are not needed to be considered. However, $n$ must be replaced by $K_{C}$ in (16). In this case, the error vectors in (15) and (16) should be removed.

Finally, for either hybrid detection or pure MMSE detection, the average BER over $\left\{h_{\hat{m}}\right\}$ can be obtained by a Monte Carlo Approach [7]. That is, by generating $N_{F}$ independent Rayleigh fading factors $\left\{h_{\hat{m}}\right\}$, the average BER can be obtained by averaging over a large number of experiments in $\left\{h_{m}\right\}$.

\section{NUMERICAL RESUlts}

In this section, some representative numerical results are presented. Unless noted otherwise, it is assumed that the number of sub-carriers $M=1024$ and the total spreading factor $N=64$. The average SNR per bit is defined as $S N R_{b}=P N /\left(2 \cdot \sigma_{n}^{2}\right)=N E_{s} /\left(2 \cdot N_{0}\right)$ and the system load is measured as $K / N$.

First of all, the BER performance of hybrid $\mathrm{MCI}$ cancellation and MMSE detection is shown in Fig. 2 for $N=N_{T} \times N_{F}=2 \times 32$ and full system load, i.e., $K / N=1.0$. A 3-stage MCI cancellor is considered. It can be seen from Fig. 2 that the hybrid detection performs much better than conventional MMSE when $S N R_{b}$ is large. The BER decreases as the number of stages increases. The performance improvement between the conventional MMSE and the hybrid detection with the $1^{\text {st }}$ stage is significant. The gap in BER between the $1^{\text {st }}$ stage and $2^{\text {nd }}$ stage is also big. However, the improvement beyond the $2^{\text {nd }}$ stage is very insignificant. In conclusion, the BER performance improvement for the hybrid detection decreases as the stages increases. Considering 2stage is sufficient.

Fig. 3 shows the BER performance as a function of system load $K / N$ for a given $S N R_{b}=8 \mathrm{~dB}$ and different number of stages. It can be seen that with various system load, the hybrid detection exhibits much better performance than the conventional MMSE. When system load is light, the $\mathrm{l}^{\text {st }}$ stage is needed. However, when the system load is heavy, considering 2-stage is necessary. This is consistent with Fig. 2.

Finally, The BER of the hybrid detection is plotted in Fig. 4 as a function of the frequency domain spreading factor for a given total spreading factor and for various values of the system load. The BER of conventional MMSE is also plotted for comparison. It can be seen that for pure MMSE, the BER decreases when $N_{F}$ increases for small $N_{F}$. This is because frequency diversity gains more than the $\mathrm{MCI}$ caused. However, for different values of $N_{F}$, the pure MMSE provides very stable performance. In other words, large $N_{F}$ does not provide frequency diversity gain at all for pure MMSE. This is because more $\mathrm{MCI}$ is caused when $N_{F}$ increases. The frequency diversity gain is cancelled out by the $\mathrm{MCl}$. However, the hybrid detection performs differently. Its BER performance improves when $N_{F}$ increases for different system load.

\section{CONCLUSIONS}

The performance of VSF-OFCDM systems with hybrid $\mathrm{MCI}$ cancellation and MMSE detection is investigated in this paper. The performance of conventional MMSE and the hybrid detection has been compared extensively. The following conclusions are drawn:

1) The hybrid detection performs much better than the conventional MMSE. But the BER performance improvement for the hybrid detection decreases as the stages increases. Considering 2-stage of MCI cancellation is sufficient.

2) For the hybrid detection, a large value of frequency domain spreading factor, $N_{F}$, is expected. However, for the conventional MMSE, the value of $N_{F}$ does not make much difference in BER performance.

\section{ACKNOWLEDGEMENT}

The authors would like to thank Dr. M. Sawahashi, Dr. K. Higuchi and Dr. H. Atarashi of NTT DoCoMo for their helpful discussions.

\section{REFERENCES}

[1] A. Chouly, A. Brajal, and S. Jourdan, "Orthogonal multicarrier techniques applied to direct sequence spread spectrum CDMA systems," Proc. IEEE GLOBECOM'93, pp. 1723-1728, Nov. 1993.

[2] N. Maeda, Y. Kishiyama, H. Atarashi, and M. Sawahashi, "Variable spreading factor-OFCDM with two dimensional spreading that prioritizes time domain spreading for forward link broadband wireless access, " in Proc. IEEE VTC2003-Spring, pp. 127-132, April 2003.

[3] M. C. C. Chan and J. C. I. Chuang, "Multicode high-speed transmission with interference cancellation for wireless personal communications," in Proc. IEEE VTC'96, vol.2, pp. 661-665, May 1996. 
[4] N. Yee and J. P. Linnarz, "Multi-carrier CDMA in indoor wireless radio networks," in Proc. IEEE Int. Symp. on Personal, Indoor and Mobile Radio Commum. (PIMRC '93), pp. 109-113, Sept. 1993.

[5] S. Kaiser, "On the performance of different detection techniques for OFDM-CDMA in fading channels," in Proc: Globecom'95, pp. 20592063, Nov. 1995
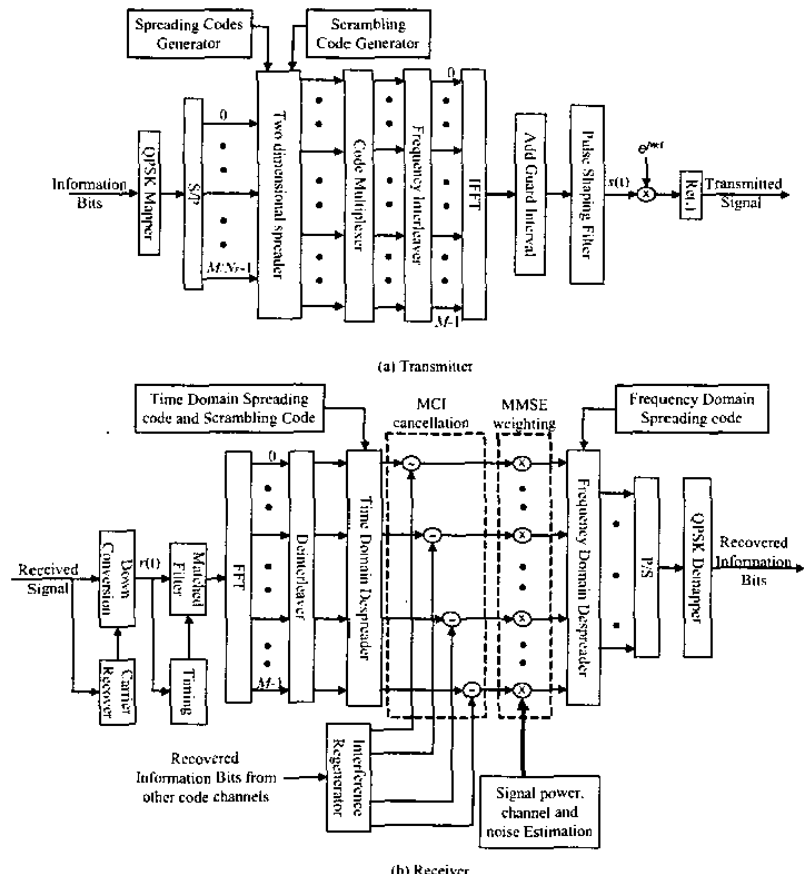

Figure 1. Block diagran for the VSF-OFCDM system with hybrid MCl canceliation and MMSE delection

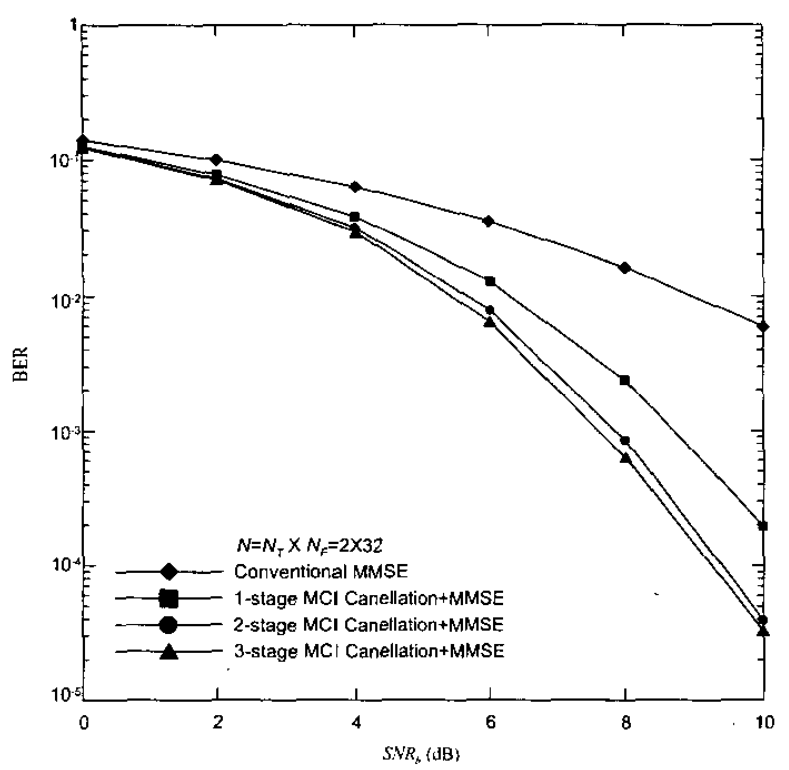

Figure 2. The performance of hybrid MCI cancellation and MMSE detection in a QPSK-modulated system with $N=2 \times 32$ and full load
[6] J. P. Linnarz, "Performance analysis of synchronous MC-CDMA in mobile rayleigh channel with both delay and Doppler spreads," IEEE Trans, on Vehicular Technology, vol. 50. no. 6, Nov. 2001.

[7] Zhou Xing, "Parallel ensemble Monte Carlo for device simulation," Workshop on High Performance' Computing Activities in Singapore. National Supercomputing Research Center, Sept. 1995.

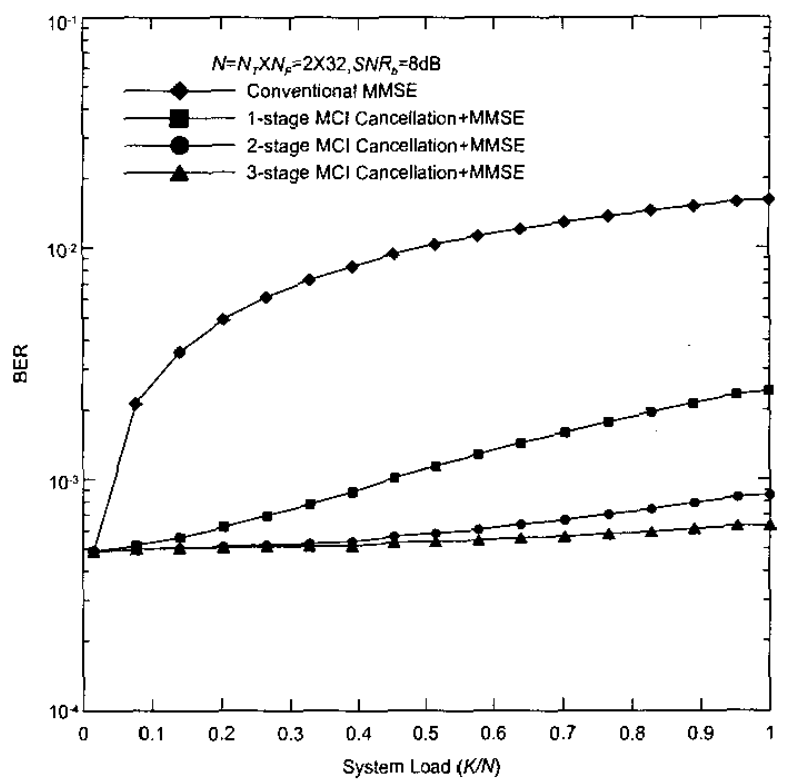

Figure 3. The performance of hybrid MCI cancellation and MMSE detection as a function of system load

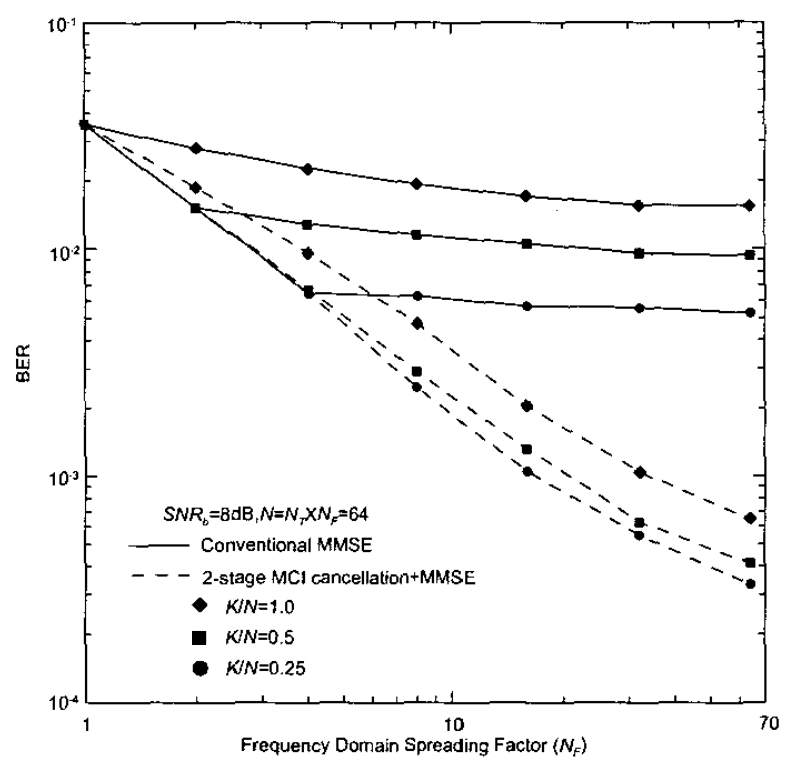

Figure 4. The system performance as a function of $N_{F}$ 